\title{
An implementation of optimal control methods (LQI, LQG, LTR) for geostationary satellite attitude control
}

\author{
Farid Djaballah $^{1}$, M.A. Si Mohammed ${ }^{2}$, Nabil Boughanmi ${ }^{3}$ \\ ${ }^{1,3}$ Department d'électronique, Université des Sciences et de la Technologied'Oran Mohamed Boudiaf, Algerie \\ ${ }^{2}$ Center of Satellite Development, Algerie
}

\section{Article Info \\ Article history: \\ Received Jun 27, 2018 \\ Revised May 4, 2019 \\ Accepted Jun 26, 2019}

\section{Keywords:}

ADCS

Geostationary satellite

KALMAN filter

LQI, LQG, LTR

Spacecraft attitude dynamics

\begin{abstract}
This paper investigates a new strategy for geostationary satellite attitude control using Linear Quadratic Gaussian (LQG), Loop Transfer Recovery (LTR), and Linear Quadratic Integral (LQI) control techniques. The sub-system satellite attitude determination and control of a geostationary satellite in the presence of external disturbances, the dynamic model of subsatellite motion is firstly established by Euler equations. During the flight mission at $35000 \mathrm{Km}$ attitude, the stability characteristics of attitude motion are analyzed with a large margin error of pointing, then a height performance-order LQI, LQG and LTR attitude controller are proposed to achieve stable control of the sub-satellite attitude, which dynamic model is linearized by using feedback linearization method. Finally, validity of the LTR order controller and the advantages over an integer order controller are examined by numerical simulation. Comparing with the corresponding integer order controller (LQI, LQG), numerical simulation results indicate that the proposed sub-satellite attitude controller based on LTR order can not only stabilize the sub-satellite attitude, but also respond faster with smaller overshoot.
\end{abstract}

Copyright @ 2019 Institute of Advanced Engineering and Science. All rights reserved.

\section{Corresponding Author:}

Farid Djaballah,

Departement d'électronique ; Laboratoire de Recherche en Système Intilligents (LARESI),

Université des Sciences et de la Technologied'Oran Mohamed Boudiaf, USTO-Mb. BP 1505,

El M'naouer, 31000 Oran, Algerie.

Email: farid.djaballah@univ-usto.dz

\section{INTRODUCTION}

The attitude control of geostationary spacecrafts [1-4], has important applications for telecommunication space missions such as pointing and for motion flying, the Attitude Determination and Control Subsystem (ADCS) provides attitude information and maintains the required spacecraft attitude during all phases of the mission, starting at spacecraft separation from the launch vehicle and throughout its operational lifetime [5-8].The selection of an ADCS is a function of many factors, Including mission objectives, satellite design, distributions torques, design constraints and stabilization required. Several control methods have been developed over the past years since the first satellite was launched. Mostly researchers all those techniques may be classified as active or passive, there are number of uncertainties that the conventional control does not account for in the design of satellite controller. The main source is the linearization of the non-linear system. These uncertainties can be adapted to a large extent by using LTR (Linear Quadratic Gaussian) compensator. The design approach for the LTR controller is based on the principle of certainty equivalence in which the Kalman filter is designed separately from the LQI optimal controller.

In the present work, we consider the problem of attitude control for rigid satellites based on the Euler representation, the performance of attitude model in state space form with LQG and LQI are examined to achieve stability for the closed-loop system in several perturbation torques acting upon a rigid 
satellite. Then the same model is simulated with LTR compensator, the simulation with LQG and LQI results, indicating an increase in robustness at higher frequency measurement perturbation. To recover the robustness of the LQI, a Loop Transfer Recovery (LTR) involves a fictitious noise source to the input of the plant in Kalman filter design. As the amplitude of injected noise becomes large in intensity, the LQG open-loop transfer function approaches that of the LQR and loop recovery takes place [9-12]

The organization of the paper is the following: mathematical preliminaries including modeling of the satellite dynamics and kinematic are presented in the next section. Section 3 presents the problem formulation and methodology including LQI, LQG and LTR. The main contribution of this paper will be presented in Section 4. Discussion and numerical simulations are also presented in Section 5. Section 6 provides the concluding remarks.

\section{PROBLEM DEFINITION AND METHODOLOGY}

Consider the spacecraft in orbit at $35000 \mathrm{Km}$, where is exposed to a several External disturbance torques, include gravity gradient, magnetic disturbances, and aerodynamic drag. To ensure the mission with height performance, it's necessary to design an attitude control system is for the case of constant attitude tracking to minimize a pointing error. The main objective and methodology of this paper is to find the optimal controller that consists of the rigid spacecraft such that asymptotic stability is achieved. Problem definition as shown in Figure 1.

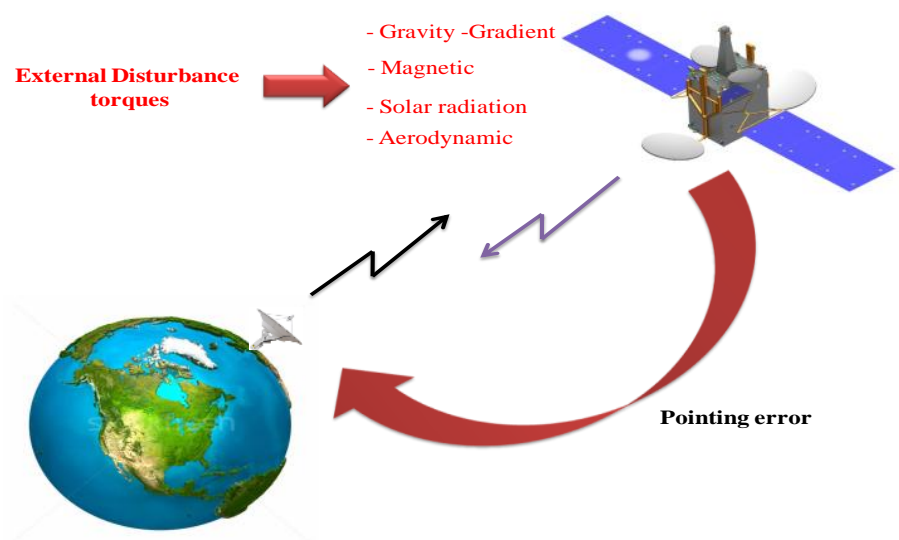

Figure 1. Problem definition

The vibrations generated by the static and dynamic forces of rotating equipment, which are generally the reaction wheels that are used for the Attitude Determination and Control Subsystem (ADCS) placed in the neighborhood of payload, are one of the most important factors affecting the pointing payloads and precision devices in space platforms. This reaction wheels has a complex configuration with gears more detail in Figure 2, which makes it has a complex vibration mechanism such as vibration coupling structure, these vibrations are caused by the speed control system of the wheels, and mainly due to the peaks of response in transitional regime [13-14].Therefore, it is necessary to analyze and minimize these peaks. On the basis of this, an implementation and an effective comparisons of three control algorithms.

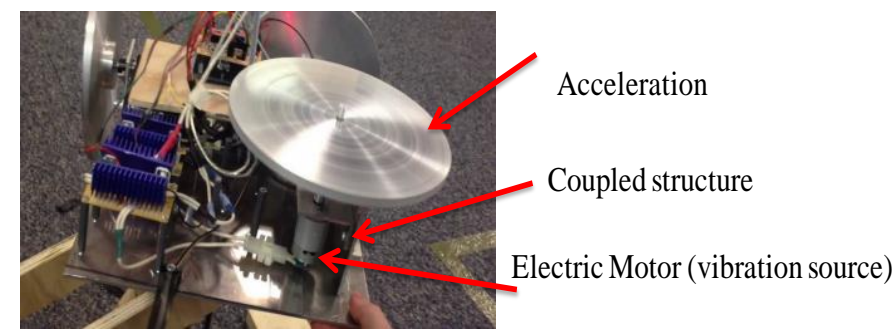

Figure 2. Micro-vibration propagation in satellite structure 
The natural frequencies and displacement configurations follow equations in [15]. For a lateral configuration, the natural frequency reads:

$$
f_{\text {lnat }}=0.560 \sqrt{\frac{E . I}{M_{\text {sat }} L^{3}}}
$$

The lateral displacement follows:

$$
\delta_{l}=0.125\left(\frac{M_{s a t} L^{3}}{E . I}\right) \cdot a_{c c}
$$

E: Young module, I: inercie of satellite, $M_{\text {sat }}$ : Mass of satellite, $L$ : Length of satellite and $a_{c c}$ : The acceleration due to the speed motor control.

\section{DYNAMIC AND KINEMATICS OF SPACECRAFT}

In this section, a rigid satellite model is presented [16-17]; a geostationary satellite is shown in Figure 3. The angles pitch, yaw and roll $(\theta, \Psi, \varphi)$ are defined respectively as a rotation around the body axes and $(p, q, r)$ are the angular rate. Figure 3 shows all reference used.

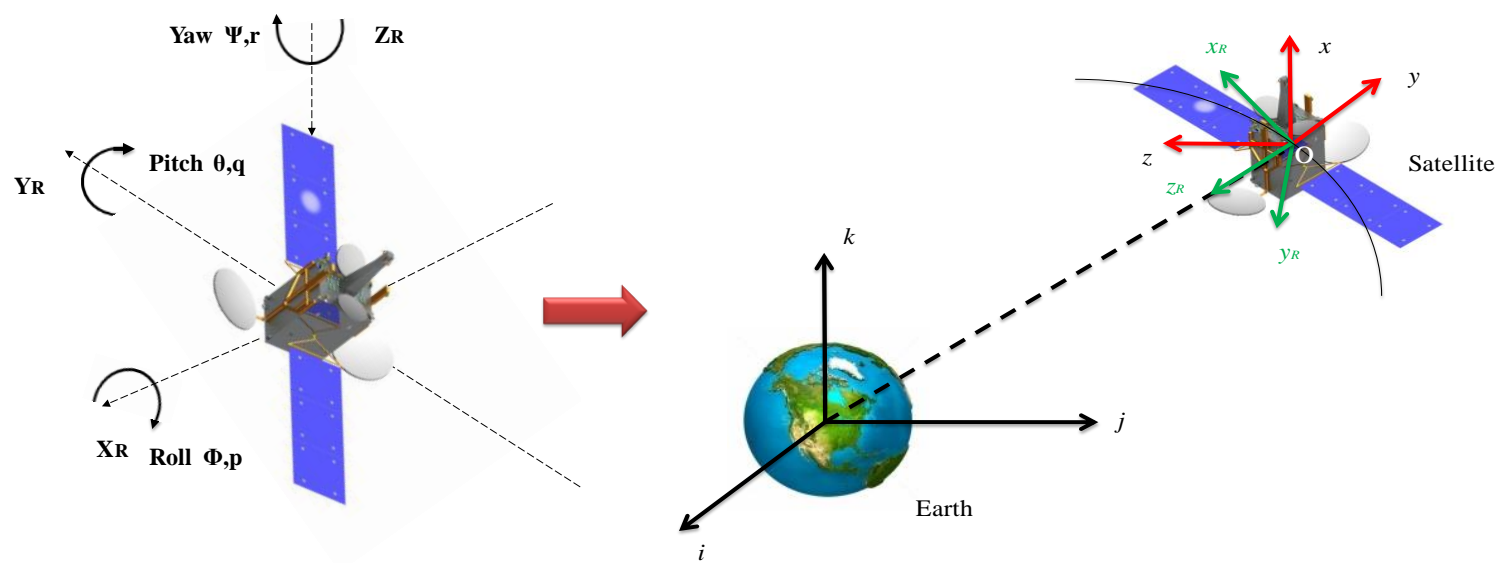

Figure 3. Frame systems (body and reference frame and inertial frame system and in motion)

\subsection{Dynamic mention}

For the rotating motion of the satellite, the moments Euler equation can be described as the followings equation:

$$
M=\frac{d H}{d t\rfloor_{I}}
$$

$\mathrm{H}$, The angular momentum of the satellite calculated in $\mathrm{O}$ and $\mathrm{M}$ the inertial resultant of the moments applied to the satellite as shown in Figure 3, then the kinetic moment of the satellite is calculated by summation of the subsystem masses of the satellite.

$$
\begin{aligned}
& \left.H=\int r \wedge V_{1} d m=\int r \wedge V_{o} d m+\int r \wedge\left(\omega_{B}\right\rfloor_{I} \wedge r\right) d m \\
& \left.H=V_{o} \wedge \int r d m+\int r \wedge\left(\omega_{B}\right\rfloor_{I} \wedge r\right) d m
\end{aligned}
$$


Assuming that $\mathrm{O}$ is the center of mass of the satellite

$$
\int r d m=0
$$

And developing the second term, we obtain in the base $(x, y, z)$

$$
H=\left[\begin{array}{ccc}
\omega_{x} \int\left(y_{i}^{2}+z_{i}^{2}\right) d m & -\omega_{y} \int y_{i} x_{i} d m & -\omega_{z} \int z_{i} x_{i} d m \\
-\omega_{x} \int y_{i} x_{i} d m & +\omega_{y} \int\left(x_{i}^{2}+z_{i}^{2}\right) d m & -\omega_{z} \int z_{i} y_{i} d m \\
-\omega_{x} \int y_{i} z_{i} d m & -\omega_{y} \int y_{i} z_{i} d m & +\omega_{z} \int\left(y_{i}^{2}+x_{i}^{2}\right) d m
\end{array}\right]
$$

Using the definition of the inertial moments of the satellite around its orthogonal axes as well as the definition of the inertial products, we obtain the following matrix formulation.

$$
\begin{aligned}
& H=I \omega_{B\rfloor I} \\
& I=\left[\begin{array}{ccc}
I_{x x} & -I_{x y} & -I_{x z} \\
-I_{x y} & I_{y y} & -I_{y z} \\
-I_{x z} & -I_{y z} & I_{z z}
\end{array}\right], \text { is the inertial matrix of the satellite }
\end{aligned}
$$

Projecting this equation on the axes of the reference B (or axes of Euler), we obtain Euler's equations:

$$
\begin{gathered}
M_{x}=\dot{H}_{x}+\omega_{y} H_{z}-\omega_{z} H_{y} \\
M_{y}=\dot{H}_{y}+\omega_{z} H_{x}-\omega_{x} H_{z} \\
M_{z}=\dot{H}_{z}+\omega_{x} H_{y}-\omega_{y} H_{x}
\end{gathered}
$$

We assuming that the axes are the main axes of inertia

$$
\begin{aligned}
H_{x}=I_{x x} \omega_{x} H_{y} & =I_{y y} \omega_{y} H_{z}=I_{z z} \omega_{z} \\
{\left[\begin{array}{c}
\omega_{x} \\
\dot{\omega}_{y} \\
\dot{\omega}_{z}
\end{array}\right] } & =\left[\begin{array}{l}
\frac{\left(I_{y y}-I_{z z}\right) \omega_{y} \omega_{z}+u_{x}+T_{x}}{I_{x x}} \\
\frac{\left(I_{z z}-I_{x x}\right) \omega_{x} \omega_{z}+u_{y}+T_{y}}{I_{y y}} \\
\frac{\left(I_{x x}-I_{y y}\right) \omega_{x} \omega_{y}+u_{z}+T_{z}}{I_{z z}}
\end{array}\right]
\end{aligned}
$$

$u\left(u_{x}, u_{y}, u_{z}\right)$ : The components of the control moment vector $\left(T_{x}, T_{y}, T_{z}\right)$ : The components of the moment vector of the exogenous disturbances.

\subsection{Kinetic mention}

For the kinetic mention, the rotation matrix can then be generated by three elementary rotations defined by the matrices: $\left(T_{\theta}, T_{\varphi}, T_{\Psi}\right)$

$$
T_{\Psi}=\left[\begin{array}{ccc}
\cos \Psi & -\sin \Psi & 0 \\
\sin \Psi & \cos \Psi & 0 \\
0 & 0 & 1
\end{array}\right], T_{\theta}=\left[\begin{array}{ccc}
\cos \theta & 0 & \sin \theta \\
0 & 1 & 0 \\
-\sin \theta & 0 & \cos \theta
\end{array}\right], T_{\varphi}=\left[\begin{array}{ccc}
1 & 0 & 0 \\
0 & \cos \varphi & -\sin \varphi \\
0 & \sin \varphi & \cos \varphi
\end{array}\right]
$$

The rotation matrix is then $T_{\varphi} T_{\theta} T_{\Psi}$

$$
T_{\varphi} T_{\theta} T_{\Psi}=\left[\begin{array}{ccc}
\cos \theta \cos \Psi & \cos \theta \sin \psi & -\sin \theta \\
-\cos \varphi \sin \Psi+\sin \varphi \sin \theta \cos \Psi & \cos \varphi \cos \Psi+\sin \varphi \sin \theta \sin \Psi & \sin \varphi \cos \theta \\
\sin \varphi \sin \Psi+\cos \varphi \sin \theta \cos \Psi & -\sin \varphi \cos \Psi+\cos \varphi \sin \theta \sin \Psi & \cos \varphi \cos \theta
\end{array}\right]
$$


The coordinates of the angular velocity vector of the reference point linked to the satellite B with respect to the reference $\mathrm{R}$ can be written as:

$$
\begin{aligned}
& p=-\dot{\Psi} \sin \theta+\dot{\varphi} \\
& q=\dot{\Psi} \cos \theta \sin \varphi+\dot{\theta} \cos \varphi \\
& r=\dot{\Psi} \cos \theta \sin \varphi+\dot{\theta} \sin \varphi
\end{aligned}
$$

Thus, by inverting the previous equation, it will always be possible to calculate the angles the Euler of the satellite in any reference.

$$
\begin{aligned}
& \dot{\varphi}=p+[q \sin \varphi+r \cos \varphi] \tan \theta \\
& \dot{\theta}=q \cos \varphi-r \sin \varphi \\
& \dot{\Psi}=[q \sin \varphi+r \cos \varphi] \cos ^{-1} \theta
\end{aligned}
$$

\section{LQI/LQR/LTR OPTIMAL CONTROLS METHODS}

A main objective of feedback system design is to achieve a nominal performance specification for a given design model of the plant, and to maintain this performance over a range of expected errors between the design model and the true plant [18-21]. In this section, three control methods are proposed and described in brief: LQI controller, LQG and LTR based on optimal control theory, the proposed approach in this paper is detailed in the scheme presented in Figure 4.

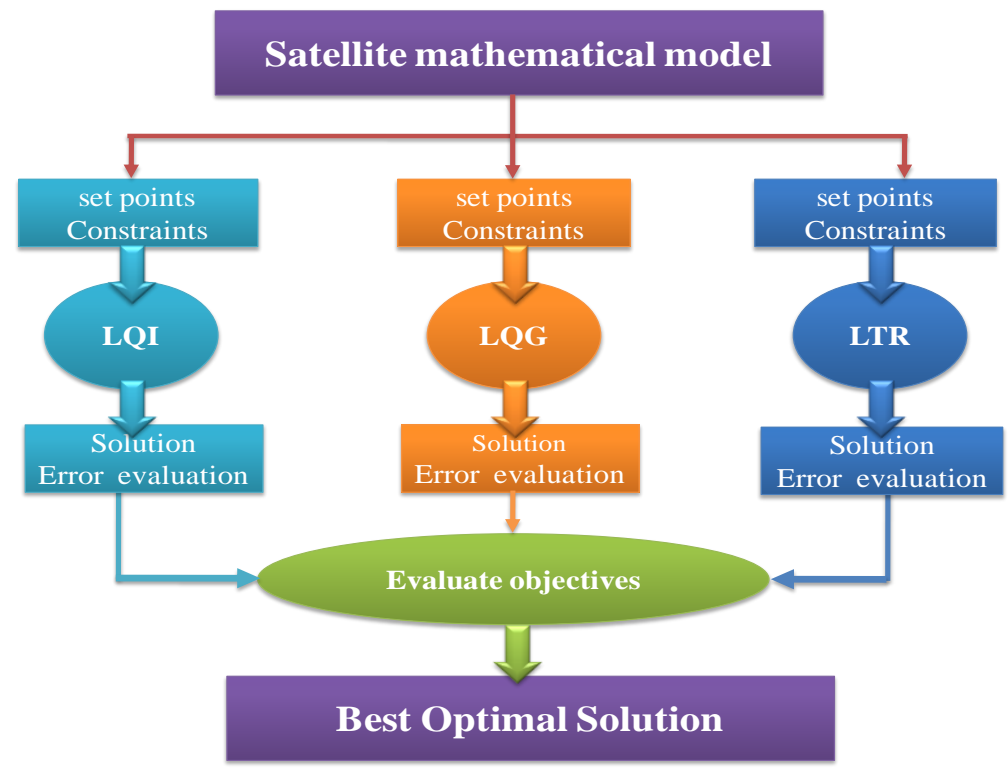

Figure 4. Optimall controllers

\subsection{LQI: Linear Quadratic with integral}

LQ I (linear quadratic with integral) controllers are the static feedback controllers based upon plant state and integral (for continuous plants) or sum (for discrete plants) of tracking error. They stabilize the outer loop $\mathrm{s}$ and minimize the quadratic cost functional associated with step exogenous inputs. Step reference input and step disturbance [22].

\subsection{LQG: linear quadratic gaussian}

The Linear-Quadratic-Gaussian (LQG) control paradigm is generally well-understood in literature. There are many methods available of calculating and minimizing the expected cost $\mathrm{E}[\mathrm{J}]$. The implementation of a LQG control design method [23] to linearized port Hamiltonian system defined by (6) to derive the dynamic observer based controller Figure 5. 


$$
\begin{aligned}
& \hat{x}=\left[(J-R) Q-B K-F B^{T} Q\right] \hat{x}+F u_{c} \\
& y_{c}=K \hat{x}
\end{aligned}
$$

Where the state of the controller $\hat{\mathrm{x}}$ represents the estimation of the state $\mathrm{x}$ of the system, the feedback gains are:

$$
\mathrm{F}=\mathrm{P}_{\mathrm{f}} \mathrm{QBR}_{\omega}^{-1}, \mathrm{~K}=\widetilde{\mathrm{R}}^{-1} \mathrm{~B}^{\mathrm{T}} \mathrm{P}_{\mathrm{c}}
$$

Where $\mathrm{P}_{\mathrm{f}}=\mathrm{P}_{\mathrm{f}}^{\mathrm{T}}>0$ are the solutions of the following Riccati equations:

$$
\begin{aligned}
& (J-R) Q_{f}+P_{f} Q(J-R)^{T}-P_{f} Q B R_{\omega}^{-1} B^{T} Q P_{f}+Q_{v}=0 \\
& Q(J-R)^{T} P_{c}+P_{c}(J-R) Q-P_{c} B \widetilde{R}^{-1} B^{T} P_{c}+\widetilde{Q}=0
\end{aligned}
$$

With $\mathrm{Q}_{\mathrm{v}}=\mathrm{Q}_{\mathrm{V}}^{\mathrm{T}}>0, \mathrm{R}_{\omega}=\mathrm{R}_{\omega}^{\mathrm{T}} \geq 0$ the covariance matrices and $\widetilde{\mathrm{Q}}=\widetilde{\mathrm{Q}}^{\mathrm{T}}>0$ and $\widetilde{\mathrm{R}}=\widetilde{\mathrm{R}}^{\mathrm{T}} \geq 0$ the optimal control weighting matrices. The cost function of the optimal control is

$$
\mathrm{J}_{c}=\int_{0}^{\infty}\left(x^{\mathrm{T}} \widetilde{\mathrm{Q}} \mathrm{x}+\mathrm{u}^{\mathrm{T}} \widetilde{\mathrm{R}} \mathrm{u}\right) \mathrm{dt}
$$

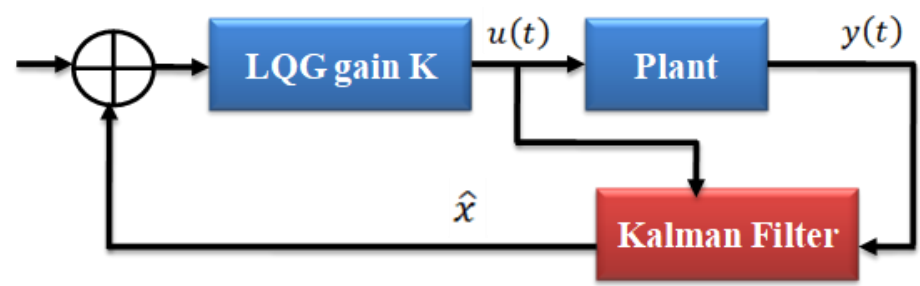

Figure 5. Close loop with kalman filter

\subsection{LTR: loop transfert recovery}

Loop Transfer Recovery is a tool applied in robust multi variable control. LTR design is the last step in a three step design procedure for constructing dynamic compensators [24]. For a $m \times n$ dimensional $G(s)$ has only minimum phase transmission zeros, then the open- loop transfer function.

$$
G(s) G_{c}(s)=\left[C(s I-A)^{-1} B\right]\left[K(s I-A+B K+F C)^{-1} F\right] \stackrel{\sigma \rightarrow 0}{\longrightarrow} C(s I-A)^{-1} F
$$

$K$ and $\sigma$ are from $L Q[(A, B)$ controlable, $(A, C)$ observable $]$

$$
\begin{aligned}
& J=\int_{0}^{\infty}\left(x^{T}(t) C^{T} C_{x}(t)+\sigma u^{T}(t) N u(t)\right) d t \\
& x=A x(t)+B u(t)
\end{aligned}
$$

\section{RESULTS AND DISCUSSION}

We present in this section the different results. In order to demonstrate the superiority of attitude stabilization controller presented in this paper, LQI, LQG and LTR as follows is compared.The results presented in this sub-section were obtained with a simulator that implements the dynamics of the satellite using MATLAB and SIMULINK. The spacecraft simulation model includes the attitude dynamics and kinematics. External disturbance torques, include gravity gradient, magnetic disturbances, and aerodynamic drag are simulated. The Figure 6 present the SIMULINK bloc developed in this study with Ixx=157; Iyy=153; Izz=157; 


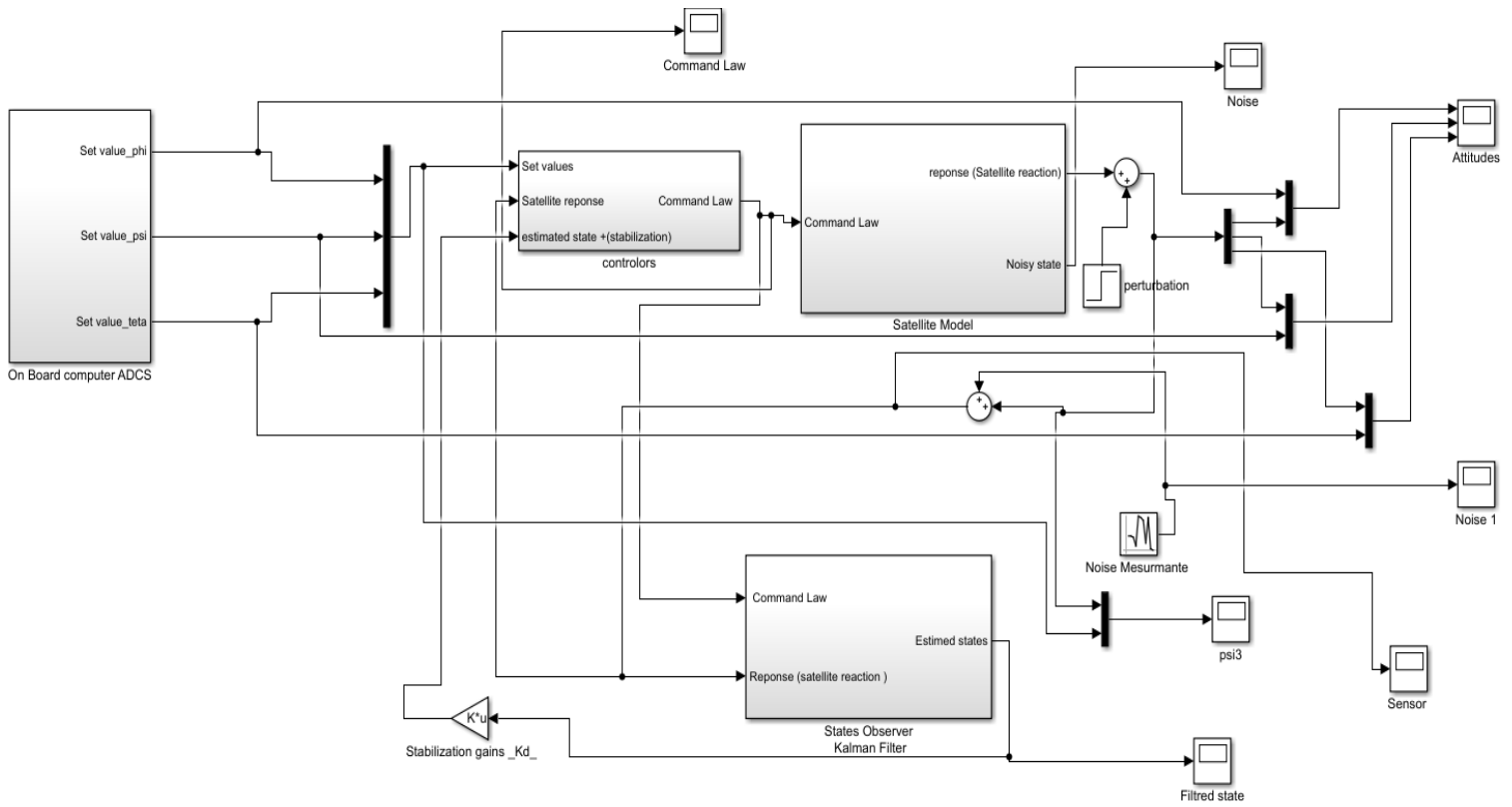

Figure 6. Simulink bloc devolloped

The A, B, C, D matrix are presented:

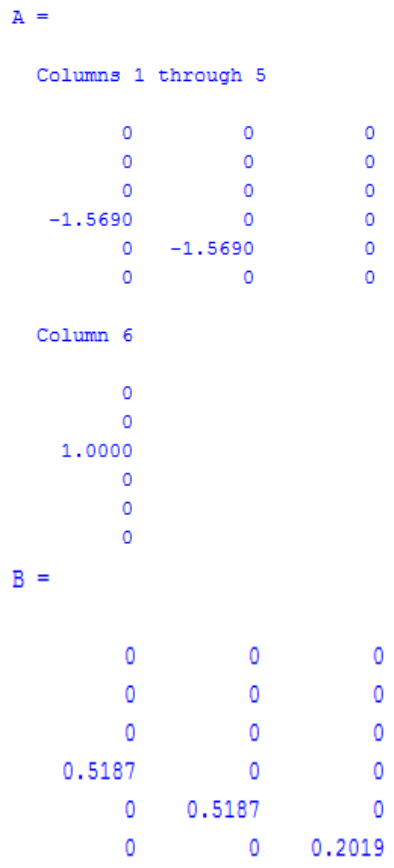

In the Figure 7, we done the simulation results in, the three angles Pitch, Yaw and Roll are plotted in spin mode in the presence of disturbance torques. The simulation results of LQI/LQG controller are given from Figure 8. Based on Figure can be found that the stabilization can not be achieved, could also be concluded as two aspects: low convergence rate and excessive initial control torque. 


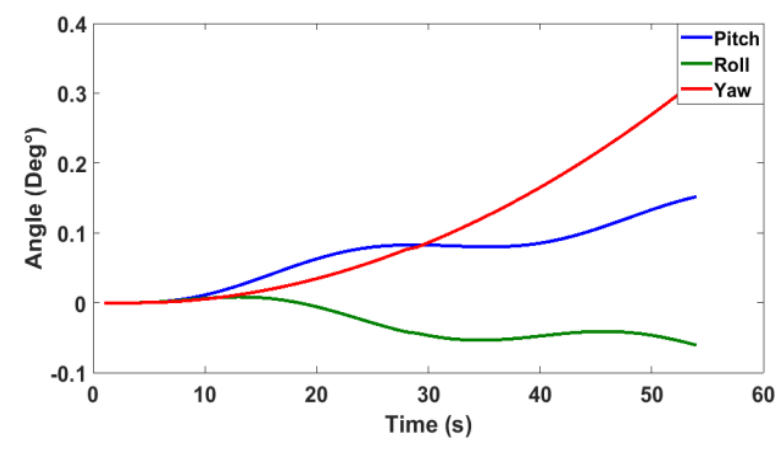

Figure 7. Spin mode of satellite

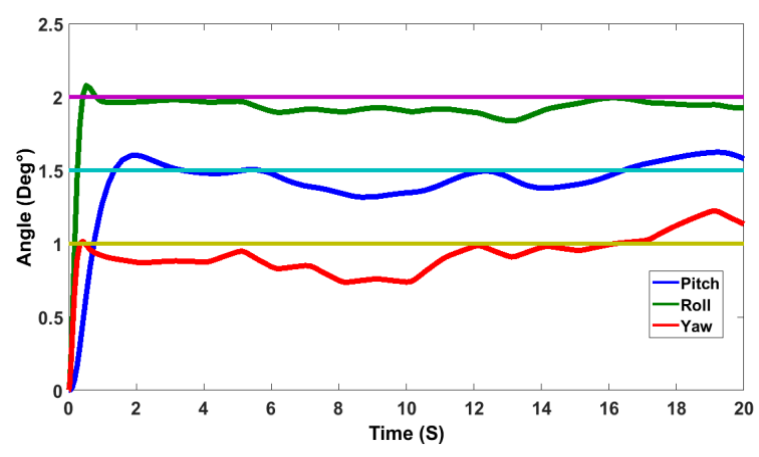

Figure 8. LQI/LQG response

The simulation results of LTR controller are given from Figure 8. Based on Figure, the performance and robustness of the LTR algorithm control for geostationnary satellite is evaluated. Based on Figure 9 it can be found that angular velocity is constant during the simulation time, and convergence rate of Eulers is maintained at a high level during this stage. This reveals that the LTR controller has a better efficiency on control torque comparing with LQI/LQG controller.

A comparative study is showed in Figure 10, the Pitch and Roll responses axes are plotted. It is observed that the angles converge within rapidly with LTR controller. The application of Response Surface Method (RSM) show in Figure 11 and 12 offers based on parameter estimates, an empirical relationship between the response variable (extraction yield of LTR) and the test variables. In Figure 11, we present the variation of lateral discplacement as function of geometry of satellite end ecceleration due to of reaction wheels speed control. We note that the discplacement increase as acceleration increase. In Figure 12, we present the variation of lateral frequency as function of geometry of satellite end the mass in Tonne. We note that the frequency varie as function of the geometry and the mass.

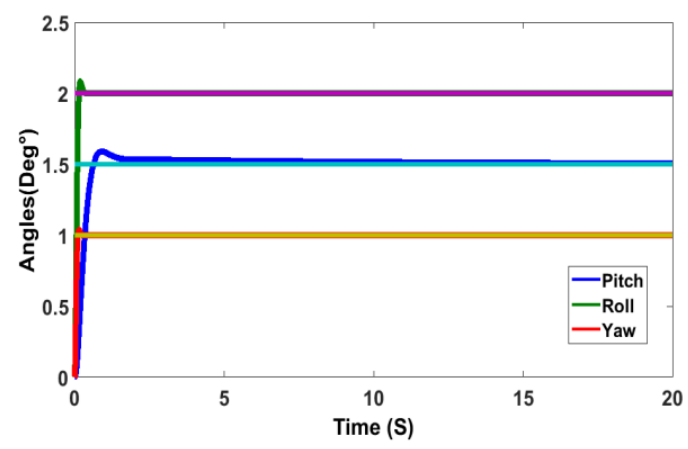

Figure 9. LTR response
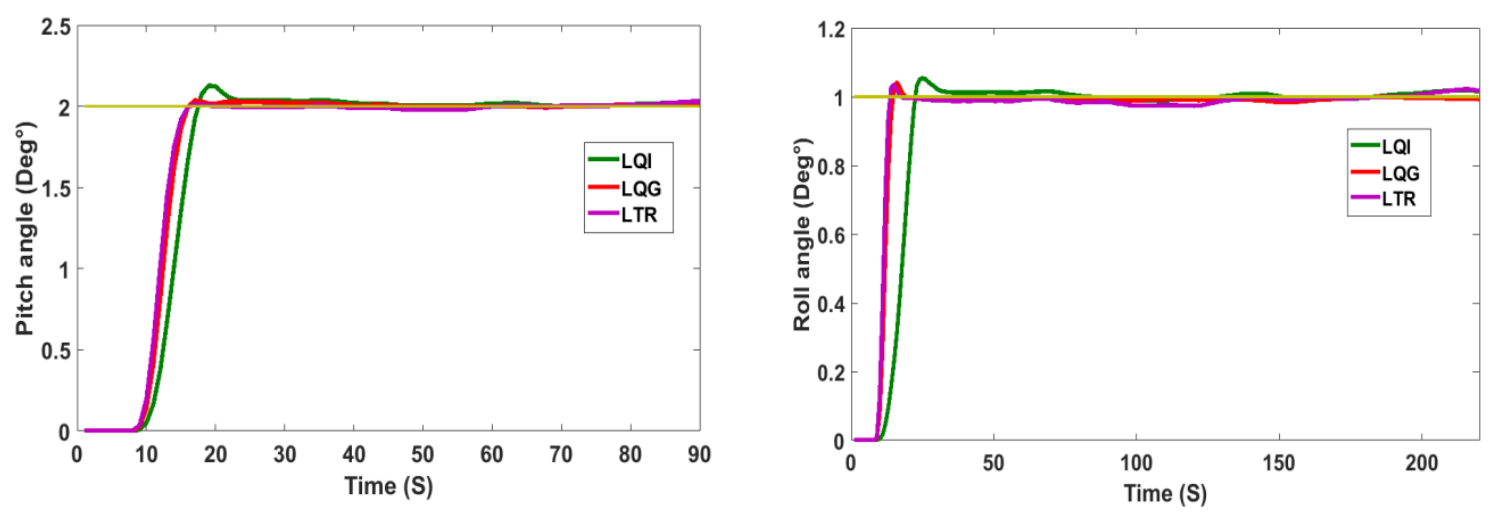

Figure 10. Angles rate LQI/LQG/LTR response 


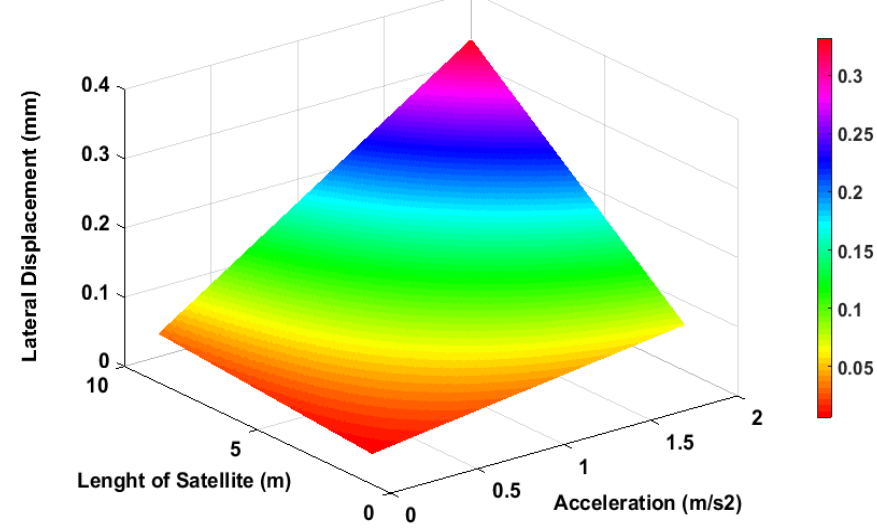

Figure 11. Response surface plots showing the effects of variables on the response (Lateral displacement)

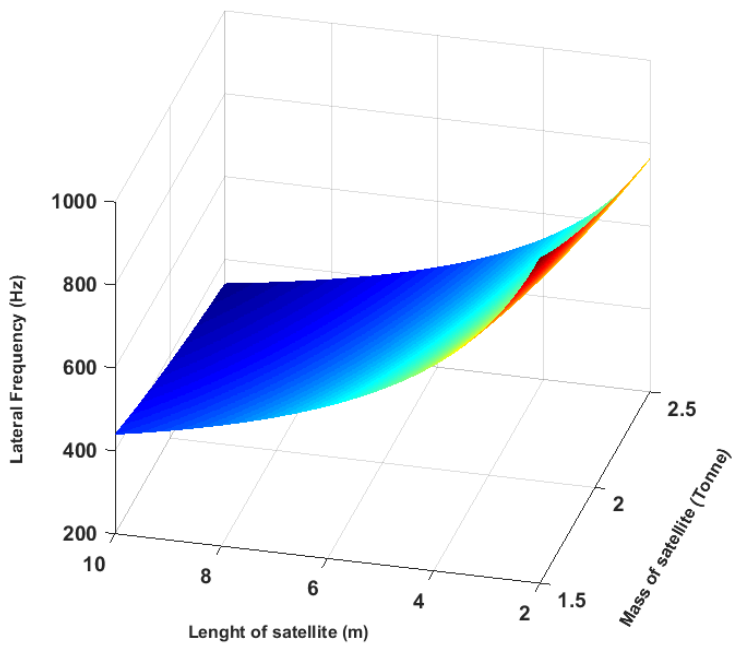

Figure 12. Response surface plots showing the effects of variables on the response (Lateral frequency)

\section{CONCLUSION}

In this paper, we analyzed the performance of a geostationary satellite stabilized, LQI, LQG and LTR controller algorithms was introduced and simulated. The three controllers was evaluated on a nonlinear system of a geostationary satellite with height inertia compared with microsatellite, proposed stability analysis for the control law of Euler's -based control system that employs saturation function has been presented. Furthermore, the Euler's based Extended Kalman Filter (EKF) analyzed in this paper was implemented. The simulation of ADCS sub-system was performed by applying different perturbation. The results showed that the LQI and LQG controller was not able to achieve stable operation in the presence of disturbance.

On the other hand, the proposed method LTR that employs EKF observer was found to effectively improve both small signal stability and transient stability of the ADCS sub-system subjected to severe disturbances.

\section{REFERENCES}

[1] S. Dhingra, D. Kumar, "A review of remotely sensed satellite image classification," International Journal of Electrical and Computer Engineering (IJECE), vol. 9, no. 3, pp. 1720-1731, Jun 2019.

[2] L.T. Phuon, B. Journet, D.B. Gia, "A microwave active filter for nanosatellite's receiver front -ends at S-band," International Journal of Electrical and Computer Engineering (IJECE), vol. 9, no. 2, pp. 973-981, Apr 2019. 
[3] E.H. Karam, N.A. Al-Awad, N.S. Abdul-Jaleel, "Design nonlinear model reference with fuzzy controller for nonlinear SISO second order systems," International Journal of Electrical and Computer Engineering (IJECE), vol. 9, no. 4, pp. 2491-2402, Aug 2019.

[4] T. Someswari, A.K. Tiwari, R. Nagaraj, "Novel Framework for Navigation using Enhanced Fuzzy Approach with Sliding Mode Controller," International Journal of Electrical and Computer Engineering (IJECE), vol. 8, no. 6, pp. 4310-4320, , December 2018.

[5] A.Yu. Aleksandrov, E.B. Aleksandrova, A.A. Tikhonov, "Stabilization of a programmed rotation mode for a satellite with electrodynamic attitude control system," Advances in Space Research, vol. 62, pp. 142-151, 2018.

[6] P. Zhang, A. Mills, J. Zambreno, P.H. Jones, "The design and integration of a software configurable and parallelizedcoprocessor architecture for LQR control," J. Parallel Distrib. Comput., vol. 106, pp. 121-131, 2017.

[7] A.Fadavi, A.Beheshti2, P.Kermani, "Offine/Online Optimum Routing of a UAV using Auxiliary Points," International Journal of Electrical and Computer Engineering (IJECE), vol. 7, no. 1, pp. 392-401, 2017.

[8] Z. Peng, L. Jikai. "On new UAV flight control system based on Kalman\& PID," IEEE Transaction, International Conference on Harbin, 2011, vol. 2, pp. 819-823.

[9] M.R. Rahimi, S. Hajighasemi, D. Sanaei, "Designing and Simulation for Vertical Moving Control of UAV System using PID, LQR and Fuzzy logic," International Journal of Electrical and Computer Engineering (IJECE), vol. 3, no. 5, pp. 651-659, 2013.

[10] F.Shabaninia, K.Jafari, "Using LQG/LTR Optimal ControlMethod to Improve Stability and Performance of Industrial Gas Turbine System,” International Scholarly Research Network, pp. 1-8, 2012.

[11] H. Bijl, J.W. Wingerden,T.B. Schön, M. Verhaegen, "Mean and variance of the LQG cost function," Automatica, vol. 67, pp. 216-223, 2016.

[12] Y. Ebihara, T. Hagiwara, M. Araki., "Sequential tuning methods of LQ/LQI controllers for multivariable systems and their application to hot strip mills," International Journal of Control, vol. 73, no. 15, pp. 1392-1404, 1999.

[13] A. Israr, "Vibration and Modal Analysis of Low Earth Orbit Satellite," HINDAWI, vol. 2014, 2014.

[14] L. Li, L. Tan, L. Kong3 ,H. Yang, D. Wang, "Flywheel micro-vibration characters of a high resolution optical satellite," JVE INTERNATIONAL LTD.JOURNAL OF VIBROENGINEERING, vol. 19, no. 6, Sep 2017.

[15] Zafrane M.A., Boudjemai A. and Boughanmi N., "Interactive design of space manufacturing systems, optimality and opportunity," Int J Interact Des Manuf, 2018.

[16] F. Pirouzmand, "Robust Model Predictive Control Based on MRAS for Satellite Attitude Control System," International Journal of Electrical and Computer Engineering (IJECE), vol. 4, no. 1, pp. 81-92, 2014.

[17] S. Rezanezhad, "Design of Fuzzy Optimized Controller for Satellite Attitude Control by Two State actuator to reduce Limit Cycle based on Takagi-Sugeno Method," International Journal of Electrical and Computer Engineering (IJECE), vol. 4, no. 2, pp. 303-313, 2014.

[18] S. Salah, M. Hadj Sadok, A.Guessou, "A Novel Neuroglial Architecture for Modelling Singular Perturbation System," International Journal of Electrical and Computer Engineering (IJECE), vol.8, no.6, pp. 4810-4822, 2018.

[19] M.A.A. Al-Mekhlafi, H. Wahid, A. Abd Azi, "Adaptive Neuro-Fuzzy Control Approach for a Single Inverted Pendulum System," International Journal of Electrical and Computer Engineering (IJECE). vol. 8, no. 5, pp. 3657-3665, 2018.

[20] S. Ruiz, J. Patino and J. Espinosa, "PI and LQR controllers for Frequency Regulation including Wind Generation," International Journal of Electrical and Computer Engineering (IJECE), vol. 8, no. 5, pp. 3711 - 3721, 2018.

[21] M. Yaich, M. Ghariani, "A Novel Technique for Tuning PI-controller in Switched Reluctance Motor Drive for Transportation Systems,"International Journal of Electrical and Computer Engineering (IJECE), vol. 8, no. 6, pp. 4272-4281, Dec 2018.

[22] F. Heng. "Design of Con tinuous and D iscrete LQI Control Systems with Stable Inner Loops," China academic journal electronic publisching house, 100721172 0620787206, 2007.

[23] Y. Wua, B. Hamroun,Y. Gorrec, B. Maschke, "Reduced order LQG control design for port Hamiltonian systems," Automatica, vol. 95, pp. 86-92, 2018.

[24] D. Horla, A. Krolikowski, "Continuous-Time Adaptive LQG/LTR Control," Proceedings of the 7th IFAC Symposium on Robust Control Design The International Federation of Automatic Control Aalborg, Denmark, Jun 2012, pp. 20-22. 